\title{
La planificación estratégica en la Dirección y Supervisión Educativa.
}

The strategic planning in the Direction and Educational Supervision.

\section{Silvia Rujano de Batista}

Universidad de Panamá, Campus Central. Facultad Ciencias de la Educación.

Departamento de Administración y Supervisión Educativa

silviarujano2016@gmail.com https://orcid.org/0000-0003-0663-5755

Págs.: 123 - 131

Recibido: 20/8/2020

Aprobado: 9/9/2020

$\underline{\text { Artículo }}$

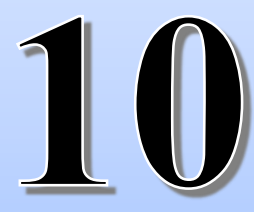

\section{Resumen}

En 1998 iniciamos la labor de supervisión educativa en una región muy empobrecida, ubicada entre los distritos de Cañazas en Veraguas y Ñurun en la Comarca Nogöbe Buglé. Es allí donde surge la necesidad de iniciar el estudio de un plan estratégico integral, sostenible y participativo entre los grupos y familias de las comunidades educativas; integradas por una población ávida de conocimientos y experiencias, capáz de contribuir con el enriquecimiento con la cultura de la escuela, la comunidad y el entorno social.La extensión educativa se inició en las 23 comunidades de la Zona de Supervisión \# 23 con el propósito de crear el Proyecto Educativo de Centro de cada escuela y comunidad, igualmente, recopilar toda la información requerida para elaborar entre todos, el Plan Estratégico Zonal, conducente a la creación del Centro de Educación Básica General Hermana María Mercedes Pérez, del Bale Ñurun, hoy convertido en una importante y exitoso Instituto. Adicional a esta conquista se logró el 
equipamiento del mismo, la consecución de 26 hectáreas de tierra y otros significativos logros educativos y sociales.

Es importante destacar que en 2001 se inició un proyecto similar en la Zona ubicada entre San Francisco y Santa Fé, y en menos de tres años, se estableció un nuevo Proyecto educativo, denominado Centro de Educación Básica General de San José \#2, y la comunidad recibió los beneficios del servicio de agua potable con su acueducto rural.

La realización de juntas de trabajo y estudio en las comunidades para investigación e interacción social, contribuyó a promover y fortalecer una conciencia comunitaria para enfrentar la solución de las necesidades mas apremiantes de sus moradores , como también mejorar la calidad de la extensión educativa, en áreas remotas y vulnerables por la condición socioeconómicas de la población .

Palabras Claves: Plan estratégico, empobrecimiento, comunidad educativa, Proyecto Educacional Centro

\begin{abstract}
In 1998 we started the work of educational supervision in a very impoverished region, located between the districts of Cañazas in Veraguas and Nurun in the Nogöbe Buglé region. It is there where the need arises to start the study of a comprehensive, sustainable and participatory strategic plan among the groups and families of the educational communities; made up of a population eager for knowledge and experiences, capable of contributing to the enrichment of the culture of the school, the community and the social environment. The educational extension began in the 23 communities of Supervision Zone \# 23 with the purpose of creating the Center Educational Project of each school and community, at the same time, compiling all the information required to develop together, the Zonal Strategic Plan, conducive to the creation of the General Basic Education Center Hermana María Mercedes Pérez, from Bale Nurun, today converted into an important and successful Institute. In addition to this conquest, the equipment was obtained, the achievement of 26 hectares of land and other significant educational and social achievements.
\end{abstract}


It is important to note that in 2001 a similar project was started in the area located between San Francisco and Santa Fé, and in less than three years, a new educational project was established, called the General Basic Education Center of San José \# 2, and the community received the benefits of the potable water service with its rural aqueduct.

The realization of work and study meetings in the communities for Research and social interaction, contributed to promoting and strengthening a community awareness to face the solution of the most pressing needs of its inhabitants, as well as improving the quality of educational extension, in areas remote and vulnerable due to the socioeconomic condition of the population.

Keywords: Strategic plan, impoverishment, educational community, Educational Project.

\section{Introducción}

En 1998 iniciamos la labor de Supervisión Educativa en una región muy empobrecida, ubicada entre los distritos de Cañaza en Veraguas y Ñurún en la Comarca Nogöbe Buglé. En nuestra mente teníamos como norte para realizar las nobles tareas asignadas al pensador y pedagogo brasileño Paulo Freire, exponente lúcido de la pedagogía crítica (Gómez, 2010) .Toda acción educativa debía nacer de la particular condición humana, de sus reales problemas sociales y culturales. Por ello, como lo expresara, Ernani María Fiori "Paulo Freire es un pensador comprometido con la vida; no piensa ideas, piensa en la existencia. Es también educador: cobra existencia su pensamiento, en una pedagogía en que el esfuerzo totalizador de la "praxis" humana busca, en la interioridad de ésta, retotalizarse como "práctica de libertad" (Freire, 1970, p.6).

De ahí nació nuestro compromiso y solidaridad con los más necesitados. En consecuencia, decidimos emprender una investigación al percatarnos de la realidad a la que nos enfrentaríamos. El ingreso de cada hogar no superaba los veinte dólares mensuales, (20.00 USD), el material de las casas era de barro y los cultivos no prosperaban en una tierra, muy pobre en nutriente necesarios para hacer producir las plantaciones y a menudo afectada por las quemas. 


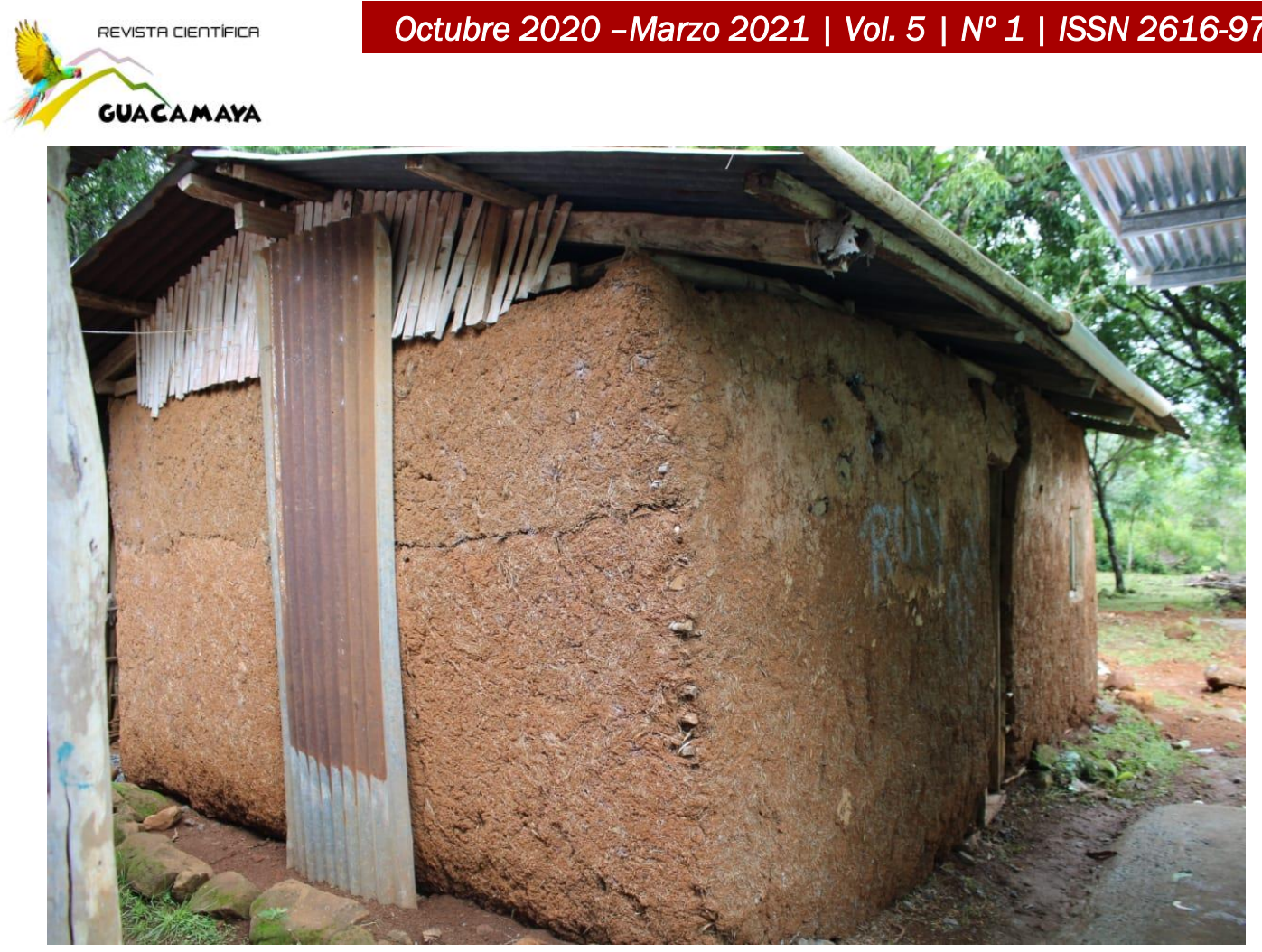

\section{Figura $\mathbf{N}^{\circ}$ 1. Las viviendas representaban el empobrecimiento de la población}

\section{ávida del Centro Educativo.}

Es allí donde surge la idea de efectuar un diagnóstico de la realidad y establecer un plan estratégico integral, sostenible y participativo, y como dice nuestra constitución la educación debe ser igual para todos sin importar las condiciones de vida y de las distancias que se encuentran los ciudadanos de los centros de enseñanza (Constitución de la República de Panamá, 1983).

Panamá, como toda América Latina, requiere de docentes, directivos y supervisores compenetrados de un gran amor por la gente, el estudio, la investigación y el trabajo educativo solidario y cooperativo, no solo en las aulas, en los ambientes virtuales, sino también entre los grupos y familias de las comunidades educativas; a menudo integradas por una población ávida de conocimientos y experiencias tendientes a enriquecer la cultura de la escuela, la comunidad y el entorno social para favorecer el desarrollo (Moorse,1998). La extensión educativa y el diagnóstico escolar se inició en las 18 comunidades de la Zona de Supervisión \# 23, con la participación organizada de los educadores, los estudiantes, instituciones, líderes y miembros de las comunidades. Este esfuerzo colectivo dio como resultado el Proyecto Educativo de Centro de cada escuela y comunidad, y permitió la recopilación de la información requerida para elaborar entre todos, del Plan Estratégico Zonal. Este modelo de 
GUACAMAYA

planificación estratégica sirvió de base a las futuras acciones llevadas a cabo para atender otras Zonas escolares.

\section{Metodología}

En la Semana de Organización del Año Escolar de 1998, reunidos todos los educadores de la Zona, con la Supervisora, se decidió, como estrategia de trabajo reuniones cada viernes de cada fin de mes sería empleado dar continuidad a la labor de revisión y control del trabajo en todas las comunidades, con el fin de darle respuesta a la amada niñez y juventud de la región, quienes terminaban su sexto grado de educación primaria y no volvían a estudiar más. Para ello realizamos giras al Ministerio de Educación, y de manera permanente a todas las Escuelas. Se organizaron seminarios de capacitación, reuniones de coordinación y de trabajo.

La grata y novedosa experiencia del trabajo en equipo se repitió en las dos Zonas siguientes.

La investigación, el estudio ,la comunión de ideas y planes entre las escuelas, comunidades e instituciones de la región, tales como el Ministerio de Desarrollo Agropecuario, el Ministerio de Educación, las Hermanas de la Presentación, Cepas Cáritas y otras instituciones, garantizó el éxito del Proyecto de Creación del Centro de Educación Básica General Hermana María Mercedes Pérez, del Bale Ñurún hoy este Centro, de manera virtual ha celebrado sus veinte años y se ha transformado en Instituto con tres Bachilleratos, Agroforestal, Comercio y Turismo. 

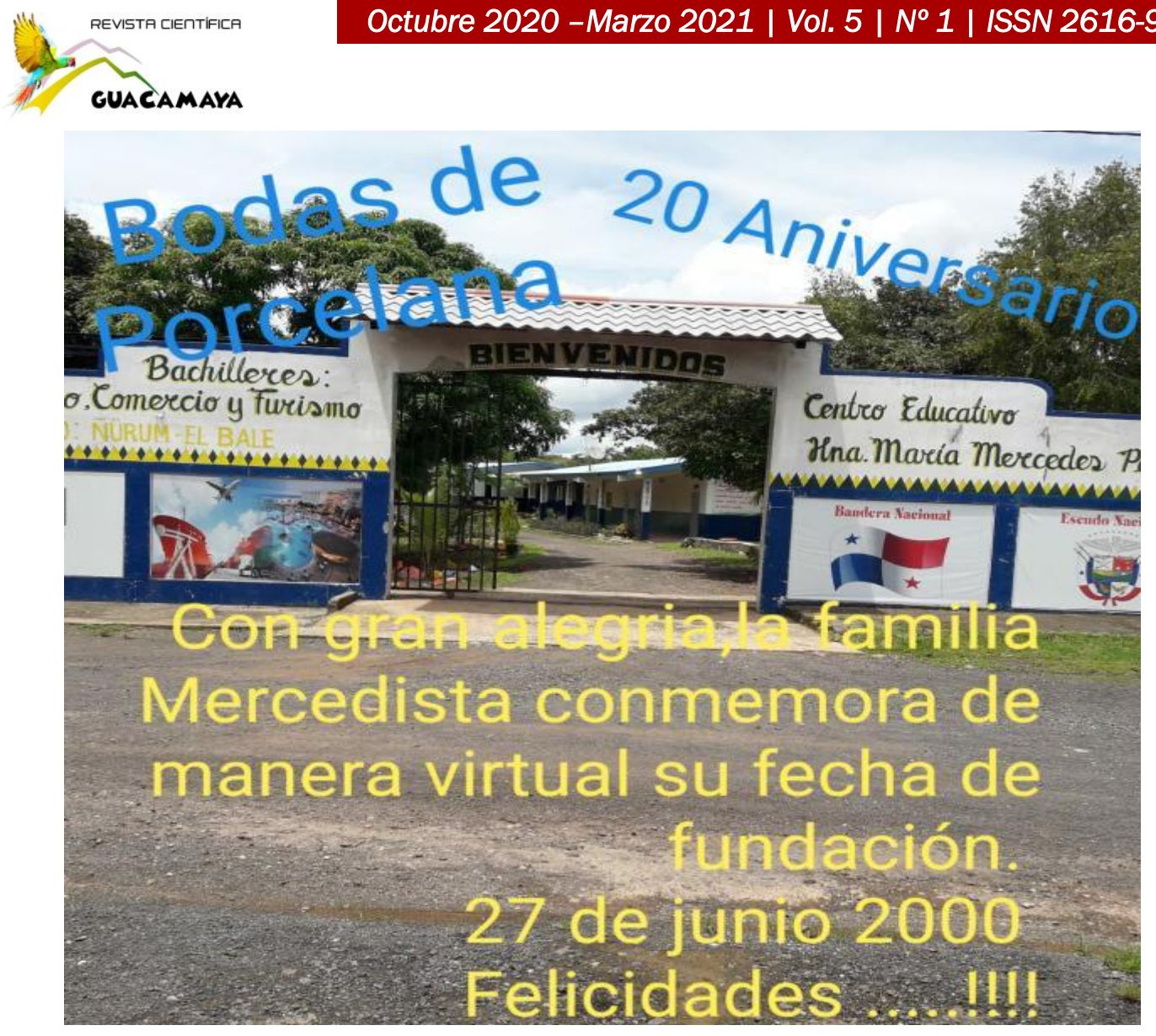

Figura $\mathbf{N}^{\circ}$ 2. Instituto Hermana María Mercedes Pérez del Bale de Nurún

\section{Resultados}

Al ser aprobado por el Ministerio de Educación, también se logró el equipamiento del mismo, con ayuda de personas e instituciones de Santiago y Panamá que creyeron en el proyecto, por ejemplo: los Padres de Familias agradecidos nos donaron 26 hectáreas de tierra que tenían de la comunidad, a orillas del río, lo que facilita el crecimiento y desarrollo del Centro educativo y proporciona tierras para la producción de cultivos. Las Hermanas de la Presentación prestaron por dos años una casa para el internado temporal de las jovencitas que llegaban a estudiar de comunidades lejanas. Vale destacar que el nombre de este gran Centro Educativo Hermana María Mercedes Pérez, se debe a que esta líder religiosa quien falleció educando y evangelizando a la población panameña en estas comunidades. 

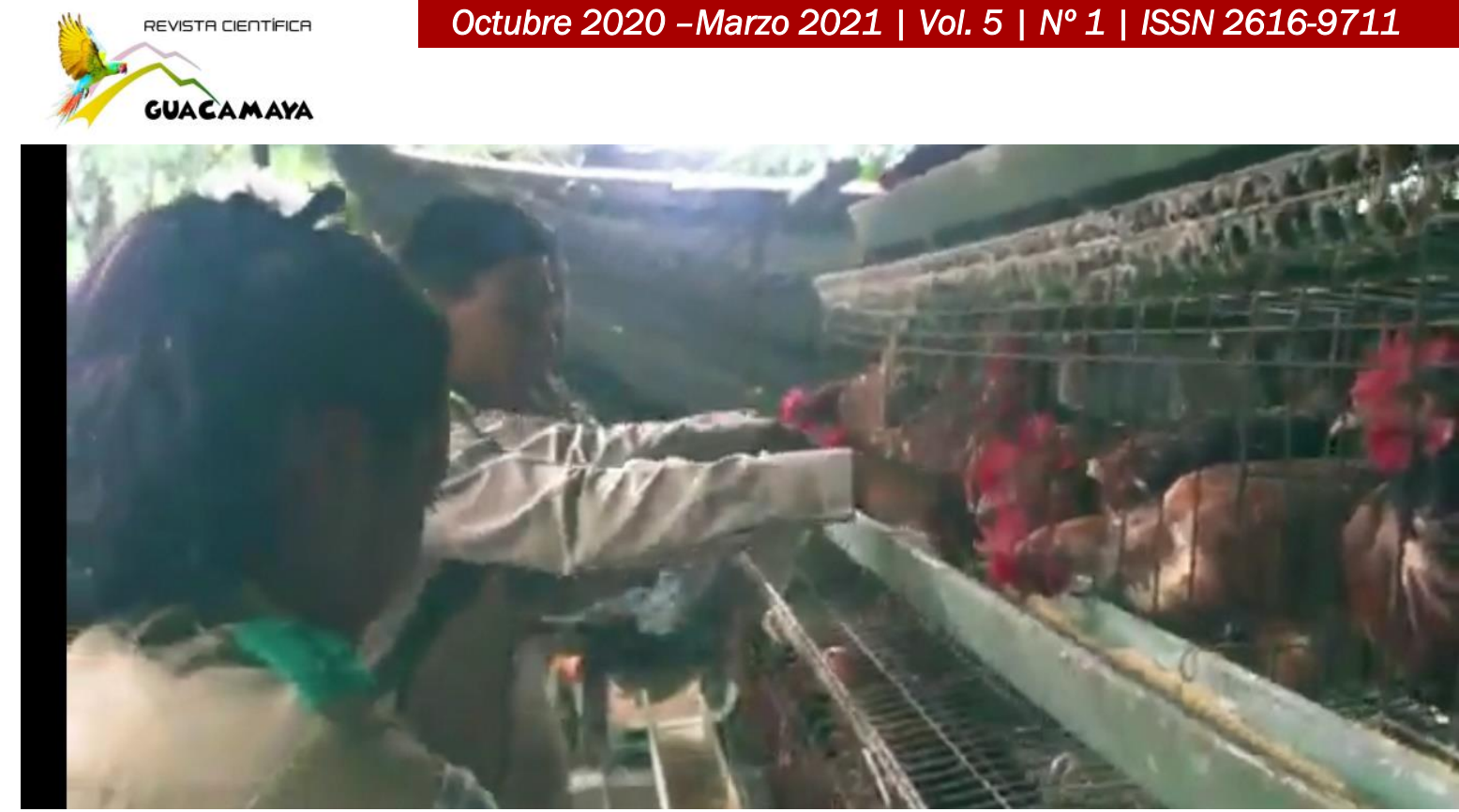

Figura $\mathbf{N}^{\circ}$ 3. Proyectos avícolas en el Instituto Hermana María Mercedes Pérez del Bale de Nurún.

Se lograron en este tiempo grandes éxitos educativos y socioeconómicos, como son los abundantes Proyectos de producción agropecuarios.

Trasladada a otra región, en 2001, también se inició un trabajo y proyecto similar en la Zona 15, ubicada entre San Francisco - Santa Fé, y en menos de tres años se logró la construcción del Proyecto educativo, Centro de Educación Básica General de San José \#”2, y el proyecto de agua para la comunidad de San José, con la participación de todas las Comunidades Educativas.

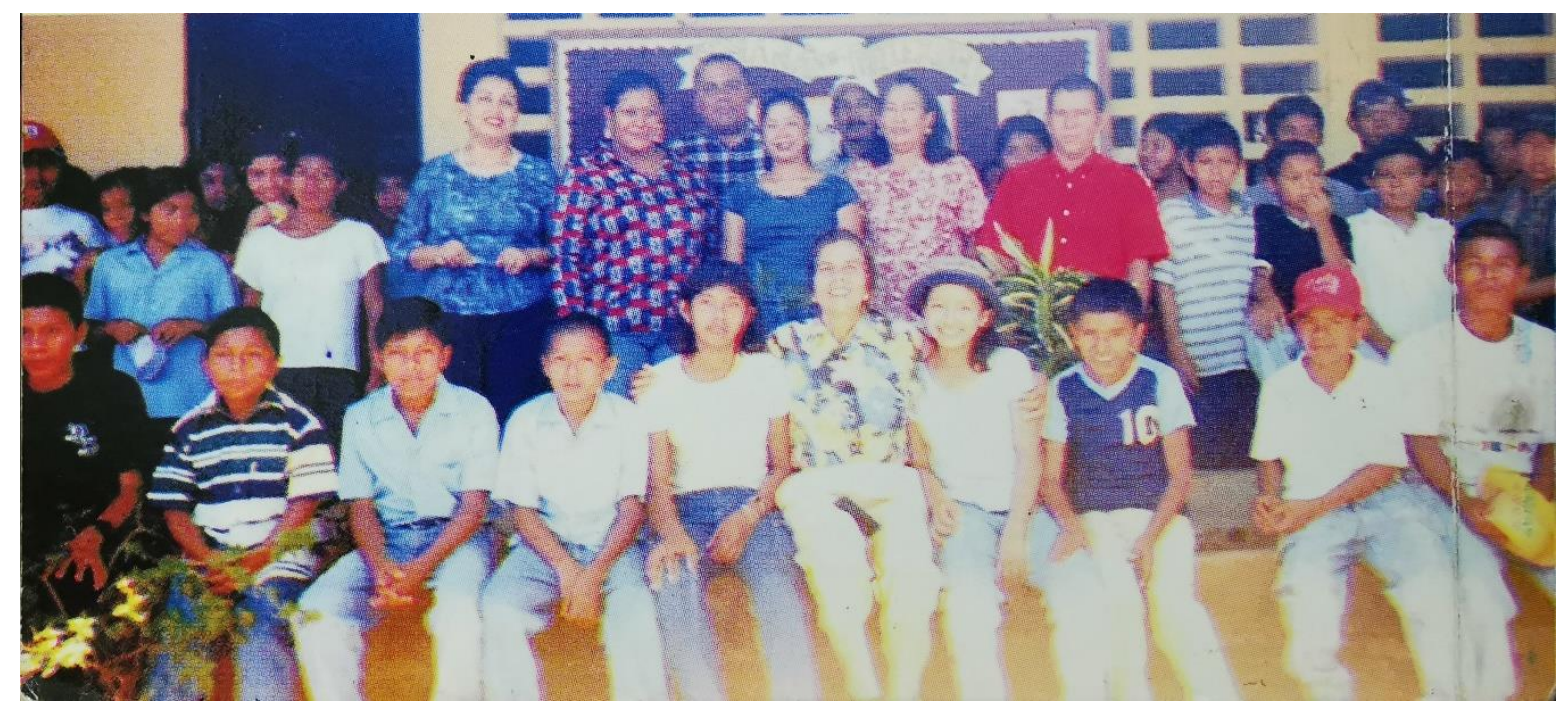

Figura $\mathrm{N}^{\circ}$ 4. La Supervisora, los Primeros Educadores y Estudiantes del Centro de Educación Básica San José \#2 


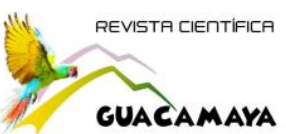

Al inicio destacamos las características de las comunidades involucradas; y después de veintidós años de iniciado el trabajo de Investigación, Planificación, Ejecución y Supervisión Zonal, nos alegra la abundante cosecha, como son los Proyectos Técnico Docentes de calidad en las aulas y fuera de ellas, los proyectos de producción agrícolas, ganaderos, hortalizas, árboles frutales y pastos.

En 2003 se nos solicitó colaborar en la Supervisión de la Zona \#31 de Ñurún, ubicada entre el Distrito de Buenos Aires y la Provincia de Bocas del Toro. Con amor aceptamos el reto, pues hace falta la entrega de los funcionarios públicos al servicio a los hermanos campesinos e Indígenas, quienes también tienen derechos a los beneficios del progreso y de la cultura de nuestro país. (Rangel, 2010). De igual manera trabajamos la investigación, planificación, organización, ejecución y controles con las Comunidades Educativas; estimulando la transformación del Centro de Educación Básica General de Buenos Aires a la categoría de Instituto.

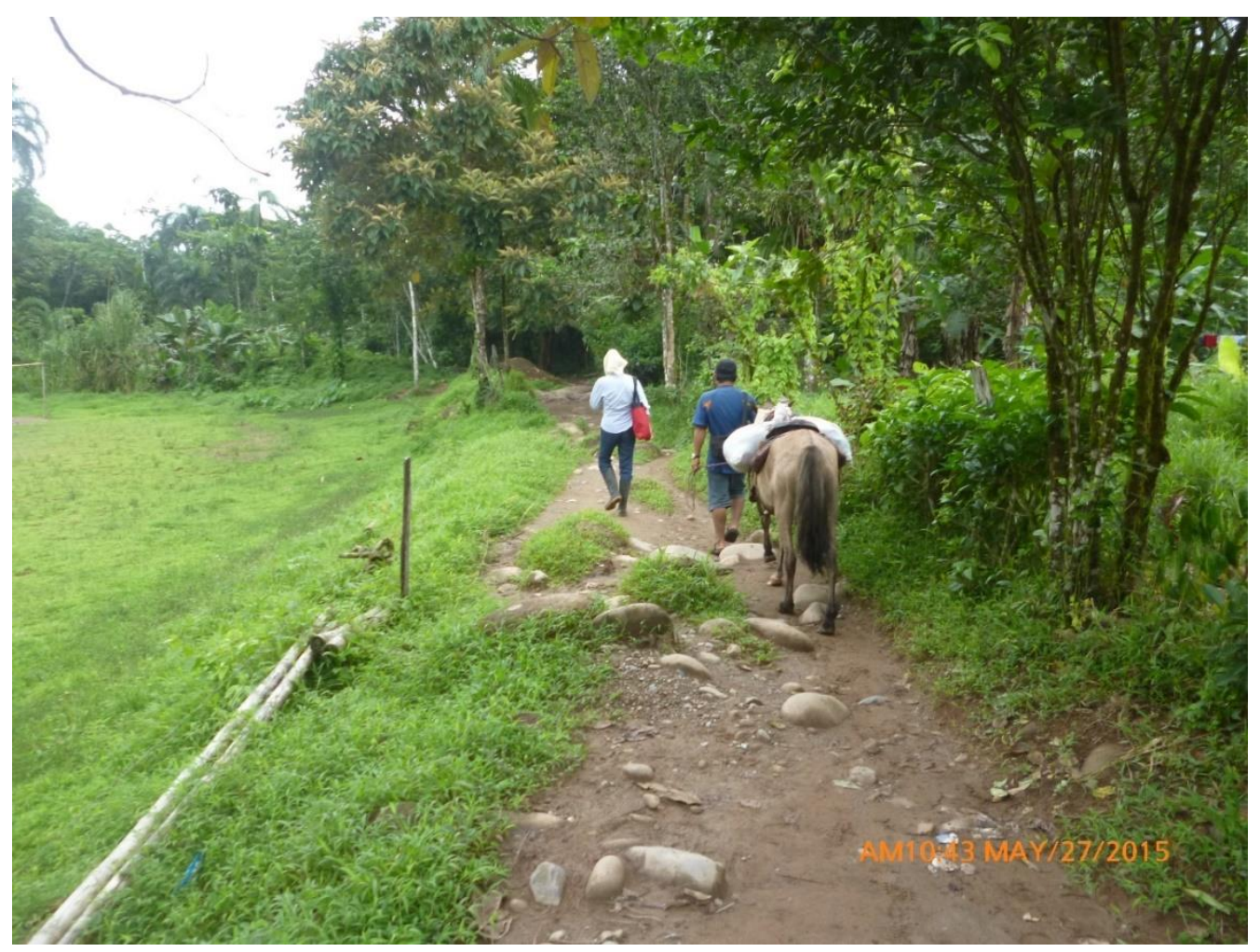

Figura N$^{\circ} 5$ Caminos de la Comarca Ngöbe Buglé, Zona de Supervisión \#31

\section{Conclusiones}

El importante trabajo de Investigación y Extensión en las tres Zonas de Supervisión ofreció la oportunidad de reconocer el gran valor de los Proyectos Educativos de Centros, P.E.C. bien elaborados con las Comunidades Educativas, estos proyectos 
siguen siendo los más importantes y bien administrados en las tres Regiones Escolares o Zonas de Supervisión. Y como han manifestado: García- Marcos, López, y Almenara (2020), en su artículo: Autorregulación del aprendizaje en la formación profesional a distancia, que: "No existe una diferencia significativa en el logro académico entre grupos". Esta realidad española se evidencia entre los amados niños y jóvenes campesinos e indígenas, cuando se les brindan las facilidades educativas mínimas, a las que tienen derecho, ellos saben aprovecharlas al máximo las oportunidades.

Se ha evidenciado también la importancia de un buen diagnóstico en la Planificación Estratégica y participativa, pues se traduce en desarrollo educativo de calidad.

Coincidimos con Manes, J.M. al reafirmar que:

"La dirección y el liderazgo son funciones de destino institucional; por lo tanto, los responsables de estas tareas, deben conocer nuevas técnicas que les permitan conducir, dirigir y mejorar proyectos educativos, eficaces desde lo pedagógico, eficientes desde lo administrativo, efectivos desde lo comunitario y trascendentes desde lo cultural." (Manes, 2003).

\section{Referencias Bibliográficas}

Constitución Nacional de la República de Panamá.(1983).

Freire, P. (1970). La Educación como práctica de la Libertad. Edit. CIRA-FAO. Chile.

García-Marcos, C., López, O., Almenara J. (2020). “Autorregulación del aprendizaje en la formación profesional a distancia. RED: Revista de Educación a Distancia.

Gómez, J. (2010). Ensayos pedagógicos. UNA,Costa Rica.

Manes, M.J. (2003). Gestión estratégica para instituciones educativas: guía para planificar estrategias de gerenciamiento institucional. Publisher: Ediciones Granica

Moorse, T.W. (1998). Introducción a la Filosofía de la Educación. Trillas.México

Ranjel, P. (2010).Desigualdad, exclusión y organismos internacionales: la invisibilización de la exclusión. Sociedad, Democracia e Identidad Centroamericana. Cuadernos Nacionales.Institutos de Estudios Nacionales. Universidad de Panamá. 